\title{
Prostaglandin E-major urinary metabolite versus fecal immunochemical occult blood test as a biomarker for patient with ulcerative colitis
}

Natsuki Ishida', Takahiro Miyazu', Ryosuke Takano', Satoshi Tamura', Shinya Tani², Takuma Kagami', Mihoko Yamade ${ }^{1}$, Yasushi Hamaya ${ }^{1}$, Moriya Iwaizumi ${ }^{3}$, Satoshi Osawa ${ }^{2}$, Takahisa Furuta ${ }^{4}$, Hiroaki Miyajima ${ }^{1}$ and Ken Sugimoto ${ }^{1 *}$

\begin{abstract}
Background: Prostaglandin E-major urinary metabolite (PGE-MUM) may be a novel biomarker for evaluating disease activity in ulcerative colitis (UC). We compared its usefulness to that of the fecal immunochemical occult blood test (FIT).

Methods: PGE-MUM and FIT measurements were performed of 92 urinary and fecal samples obtained from 60 patients with UC. Endoscopic activity was determined by Mayo endoscopic subscore (eMayo) and Ulcerative Colitis Endoscopic Index of Severity (UCEIS) score.

Results: PGE-MUM levels and FIT results showed a significant correlation with respect to eMayo $(P<0.001$ and $P<$ 0.001 , respectively), and there was a significant difference in PGE-MUM values between the groups below eMayo1 and above eMayo2 $(P=0.012)$. Both biomarkers were significantly correlated with the UCEIS score $(P<0.001$ and $P<0.001$, respectively), and the PGE-MUM values were significantly different between groups below UCEIS1 and above UCEIS2 ( $P=0.012$ ). PGE-MUM and FIT were significantly correlated with eMayo in the group with a disease duration $<5$ years $(P=0.041$ and $P<0.001$, respectively). Although PGE-MUM and eMayo differed significantly between groups over 5 years $(P=0.012)$, FIT was not correlated with eMayo $(P=0.101)$.

Conclusions: PGE-MUM is useful as a biomarker as FIT for evaluating the endoscopic activity, particularly in longterm affected patients with UC.
\end{abstract}

Keywords: Ulcerative colitis, Inflammatory bowel disease, Prostaglandin E-major urinary metabolite, Fecal immunochemical occult blood test

\footnotetext{
* Correspondence: sugimken@hama-med.ac.jp

${ }^{1}$ First Department of Medicine, Hamamatsu University School of Medicine,

1-20-1 Handayama, Higashi-ku, Hamamatsu 431-3192, Japan

Full list of author information is available at the end of the article
}

(c) The Author(s). 2020 Open Access This article is licensed under a Creative Commons Attribution 4.0 International License, which permits use, sharing, adaptation, distribution and reproduction in any medium or format, as long as you give appropriate credit to the original author(s) and the source, provide a link to the Creative Commons licence, and indicate if changes were made. The images or other third party material in this article are included in the article's Creative Commons licence, unless indicated otherwise in a credit line to the material. If material is not included in the article's Creative Commons licence and your intended use is not permitted by statutory regulation or exceeds the permitted use, you will need to obtain permission directly from the copyright holder. To view a copy of this licence, visit http://creativecommons.org/licenses/by/4.0/ The Creative Commons Public Domain Dedication waiver (http://creativecommons.org/publicdomain/zero/1.0/) applies to the data made available in this article, unless otherwise stated in a credit line to the data. 


\section{Background}

Ulcerative colitis (UC) is an idiopathic chronic inflammatory disorder characterized by diarrhea, bloody stool, abdominal pain, fever, anemia, and weight loss [1], and its clinical course is characteristic of repeating remission and relapse [2]. Although assessing the disease activity and response to treatment is important to achieving better disease control, current studies have recommended that achieving mucosal healing is the treatment goal of UC since it is associated with sustained clinical remission, decreased hospitalization rates, and the avoidance of colectomy [3]. Endoscopic evaluations are important because patients with UC can be asymptomatic despite the presence of mucosal inflammation. However, colonoscopy is expensive and invasive, making frequent examinations difficult. Therefore, non-invasive biomarkers of mucosal status with accuracy comparable to that of endoscopy are needed.

Serum C-reactive protein (CRP) is the most common biomarker used to assess inflammation in patients with IBD. However, some reports have stated that CRP levels may remain within the physiological range or only show mild abnormalities even in patients with endoscopically active UC [4]. Fecal calprotectin (FC) and the fecal immunochemical occult blood test (FIT) are minimally invasive techniques that reflect the endoscopic severity of UC [5-8]. However, fecal samples can be more difficult to obtain than blood or urine samples because patients are reluctant to bring fecal samples to the hospital and it is difficult to collect fecal sample from diarrhea stool. However, these problems can be circumvented by examinations using urine samples. Arai et al. reported that prostaglandin E-major urinary metabolite (PGE-MUM) was a reliable biomarker of the colonoscopic and histological appearance of UC, suggesting that it was more sensitive than those previously utilized to evaluate UC-related mucosal inflammation [9]. Here we investigated the usefulness of PGE-MUM versus FIT as a biomarker for evaluating the endoscopic activity of patients with UC.

\section{Methods}

\section{Patients and study design}

Sixty patients with UC treated at Hamamatsu University School of Medicine between August 2016 and August 2018 were included in this study. Colonoscopy was performed a total of 92 times for these 60 patients. In this study, almost all the patients received laxative administration on the day before colonoscopy. Each time, urine and fecal specimens were collected; PGE-MUM and FIT were measured; and endoscopic severity, clinical activity, and blood test results were prospectively evaluated. The diagnosis of UC was based on typical medical history and clinical features on endoscopic and histological assessments according to current guidelines. UC is a chronic inflammatory condition with a relapsing and remitting course. Patients who were not diagnosed with UC, such as those with indeterminate colitis or unclassified inflammatory bowel disease, were excluded. Smokers were also excluded since smoking can reportedly increase PGEMUM values [10]. Since the complication of chronic fibrosing interstitial pneumonia increases PGE-MUM values, patients with interstitial pneumonia were also excluded [11].

\section{Disease assessment}

Clinical disease activity was evaluated using Lichtiger's clinical activity index (CAI), which is based on the following criteria: diarrhea (number of daily stools), nocturnal diarrhea, visible blood in stool (percentage of movements), fecal incontinence, abdominal pain or cramping, general well-being, abdominal tenderness, and need for anti-diarrheal drugs [12]. Total scores range from 0 to 21 points. CAI was evaluated on the same day as the endoscopic examination, and clinical remission was defined as a $\mathrm{CAI} \leq 4$.

Patients underwent colonoscopy after performing bowel preparation consisting of a polyethylene glycol-based electrolyte solution or glycerin enema. UC mucosal status was assessed using the Mayo endoscopic subscore (eMayo) classification system and the Ulcerative Colitis Endoscopic Index of Severity (UCEIS) score [13, 14]. The eMayo scores were as follows: 0 , normal or inactive disease; 1 , mild disease with erythema, decreased vascular pattern, mild friability; 2, moderate disease with marked erythema, absence of vascular patterns, friability, erosions; and 3, severe disease with spontaneous bleeding, ulceration. The UCEIS scores were calculated as the simple sum of three descriptors: vascular pattern (score 0-2), bleeding (score $0-3$ ), and erosions and ulcers (score $0-3$ ). These were evaluated at the most active lesion within the colon. Endoscopic remission and mucosal healing were defined as eMayo scores of 0 or 1 and UCEIS scores of 0 or 1. As PGE-MUM reflects the inflammation status of the whole colon, we investigated the correlation of PGEMUM or FIT to the sum of the eMayo calculated from six segments (the cecum, ascending colon, transverse colon, descending colon, sigmoid colon, and rectum).

\section{PGE-MUM analysis}

Urine samples were obtained on the morning of the endoscopic examination at our hospital and sent to the SRL Hachioji Laboratory (Tokyo, Japan). Each spot urine sample was measured by a -counter (Hitachi) using a Bicylic PGE-MUM RIA Kit (Fuji Rebio, Tokyo, Japan). The measured PGE-MUM values were corrected with urinary creatinine. 


\section{Fecal immunochemical test analysis}

Fecal samples were prepared on the day before or of the colonoscopy to prevent bleeding due to the endoscopic examination. Patients collected a stool specimen by using a collection kit (Eiken Chemical, Tokyo, Japan). The submitted samples were immediately processed and examined using OC Sensor io (Eiken Chemical). The FIT results were obtained on the day of or within 1 week of colonoscopy.

\section{Statistical analysis}

Patient demographics and baseline characteristics were summarized using descriptive statistics. Correlations between two biomarkers (PGE-MUM and FIT) and other values or activity index values were analyzed with logistic regression analysis. Intergroup differences were compared using Student's t test. Statistical analyses of the data were performed using the Excel statistical software package (Ekuseru-Toukei 2010; Social Survey Research Information Co., Ltd., Tokyo, Japan) and the statistical program R (http://cran.r-project.org).

\section{Ethical statement}

Informed consent was obtained from all patients after explanation of the purpose of the study and the nature of the procedures involved. The study protocol was reviewed and approved by the Institutional Review Board of Hamamatsu University School of Medicine (number 18-228). Further, the investigation was conducted in accordance with Good Clinical Practice principles in adherence to the Declaration of Helsinki.

\section{Results}

\section{Patient characteristics}

The patients' baseline characteristics are shown in Table 1. Urinary and fecal samples (92 specimens each) were collected from the 60 patients with UC. The mean patient age was 48.5 years (Table 1). Mean disease duration was 7.2 years (range, $0.1-26$ ). With regard to disease extent, $57(63.0 \%)$ patients had extensive colitis, 24 (26.1\%) had left colitis, and 11 (10.9\%) had proctitis. Regarding the treatment received at the time of sampling, 58 (63.0\%) patients were taking oral 5-aminosalicylic acid (5ASA), 12 (13.0\%) were taking suppository 5-ASA, 12 (13.0\%) were taking systematic steroids, 35 (38.0\%) were taking immunomodulators, 10 (10.9\%) were taking tacrolimus, and $25(27.2 \%)$ were taking anti-tumor necrosis factor-alpha therapy. The mean PGE-MUM and FIT levels were $29.2 \mu \mathrm{g} / \mathrm{g} . \mathrm{Cr}$ and $2178 \mathrm{ng} / \mathrm{mL}$, respectively.

\section{Correlation between urinary/fecal biomarkers and blood biomarkers in patients with UC}

We next investigated the correlation between PGE-MUM and the blood biomarkers CRP and erythrocyte sedimentation rate (ESR), which represented the inflammatory condition of UC, and the blood biomarker serum albumin (Alb), which represented nutritional condition. Although PGE-MUM showed no significant correlation with CRP and ESR, PGE-MUM showed a significant negative correlation with Alb $(P=0.002)$ (Fig. 1a-c). We also investigated whether FIT was significantly correlated with CRP, ESR, and Alb. Although FIT showed no significant correlation with CRP or ESR, there was a significant negative correlation between FIT and Alb $(P=0.032)$ (Fig. 1d-f).

\section{Correlation between urinary/fecal biomarkers and endoscopic activity index in patients with UC}

All patients in this study underwent colonoscopy as well as evaluations of endoscopic severity using eMayo and UCEIS. We initially examined the correlation between PGE-MUM and eMayo and between FIT and eMayo. PGE-MUM showed a significant positive correlation with eMayo $(r=$ $0.355, P<0.001$ ) (Fig. 2a); a similarly significant positive correlation was shown in FIT $(r=0.447, P<0.001)$ (Fig.

Table 1 Demographic features of a total of 92 patients with UC

\begin{tabular}{|c|c|}
\hline Characteristics & $\boldsymbol{N}=92$ \\
\hline Age(year), mean(range) $\pm S D$ & $48.5(14-83) \pm 16.3$ \\
\hline Male/Female, n(\%) & $64 / 28(69.6 / 30.4)$ \\
\hline Disease duration(year), mean(range) \pm SD & $7.2(0.1-26) \pm 7.1$ \\
\hline \multicolumn{2}{|l|}{ Disease extent, n(\%) } \\
\hline Extensive colitis & $57(63.0)$ \\
\hline Left sided colitis & $24(26.1)$ \\
\hline Proctitis & $11(10.9)$ \\
\hline CAl(Lichtiger socre) mean(range) \pm SD & $3.4 \pm 4.3$ \\
\hline eMayo mean(range) $\pm S D$ & $1.0(0-3) \pm 1.0$ \\
\hline eMayo 0, n (\%) & $38(41.3)$ \\
\hline eMayo 1, n (\%) & $21(22.8)$ \\
\hline eMayo 2, n (\%) & $27(29.3)$ \\
\hline eMayo 3, n (\%) & $6(6.6)$ \\
\hline UCEIS mean(range) $\pm S D$ & $2.1(0-7) \pm 1.9$ \\
\hline $\begin{array}{l}\text { PGE-MUM }\left(\mu \cdot g^{-1} \cdot \mathrm{Cr}^{-1}\right) \\
\text { mean(range) } \pm S D\end{array}$ & $29.2(5.1-93.9) \pm 18.5$ \\
\hline $\begin{array}{l}\text { Fecal hemoglobin concentrations } \\
(\mathrm{ng} / \mathrm{ml}), \mathrm{n}(\%) \text { mean(range) } \pm \mathrm{SD}\end{array}$ & $2178(0-26500) \pm 4667$ \\
\hline \multicolumn{2}{|l|}{ Drug at study, n (\%) } \\
\hline Oral 5-ASA & $58(63.0)$ \\
\hline Suppository 5-ASA & $12(13.0)$ \\
\hline Systemic steroids & $12(13.0)$ \\
\hline Azathioprine / Mercaptopurine & $35(38.0)$ \\
\hline Tacrolimus & $10(10.9)$ \\
\hline Biologics & $25(27.2)$ \\
\hline
\end{tabular}

CAl clinical activity index, eMayo Mayo endoscopic subscore, UCEIS Ulcerative Colitis Endoscopic Index of severity, PGE-MUM Prostaglandin E-Major Urinary Metabolite, 5-ASA 5-aminosalicylic acid 
2b). Next, we defined eMayo 0 or 1 as endoscopic remission and eMayo 2 or 3 as endoscopically active and investigated whether PGE-MUM and FIT differed between endoscopic remission and endoscopic activity. PGE-MUM was significantly higher in patients with an eMayo of 2 or 3 than in those with an eMayo of 1 or $2(P=0.012)$ (Fig. 2c). FIT was also significantly higher in patients with an eMayo of 2 or 3 than in those with an eMayo of 1 or $2(P<0.001)$ (Fig. 2d). The PGE-MUM or FIT values showed a significant correlation with the sum of eMayo $(r=0.413, P<$ 0.001 and $r=0.390, P<0.001$, respectively; Fig. 2e and f). Regarding the correlation to PGE-MUM, the correlation coefficient of the sum of eMayo was slightly larger than the maximum eMayo $(r=0.355$ vs $r=0.413)$.

Next, we examined the correlation between PGEMUM and UCEIS score and that between FIT and UCEIS score. PGE-MUM showed a significant positive correlation with UCEIS score $(r=0.352, \quad P<0.001)$ (Fig. 3a), while FIT and UCEIS score also showed a significant positive correlation $(r=0.375, P<0.001)$ (Fig. $3 \mathrm{~b})$. Next, we defined UCEIS score $\leq 1$ as endoscopic remission and UCEIS score $\geq 2$ as endoscopic activity and investigated whether PGE-MUM and FIT differed between endoscopic remission and endoscopic activity. PGE-MUM level was significantly higher in patients with a UCEIS score $\geq 2$ than in those with a UCEIS score $\leq 1$ $(P=0.022)$ (Fig. 3c). FIT was also significantly higher in patients with a UCEIS score $\geq 2$ than in those with a UCEIS score $\leq 1(P<0.001)$ (Fig. 3d).
Next, the accuracies of PGE-MUM and FIT were assessed using receiver-operating characteristic (ROC) curves, defining an eMayo of 0 or 1 as an endoscopic remission of UC (Table 2). The area under the curve (AUC) of PGE-MUM and FIT were 0.644 (95\% confidence interval $[\mathrm{CI}], 0.523-0.76)$ and 0.862 (95\% CI, 0.74-0.912), respectively. The optimal cutoff PGE-MUM value based on eMayo was $30.9 \mu \mathrm{g} / \mathrm{g}$.Cr. Likewise, the AUC for PGE-MUM and FIT for predicting endoscopic remission by the UCEIS score were 0.636 (95\% CI, $0.522-0.765)$ and 0.789 (95\% CI, 0.698-0.879), respectively. The best cutoff for reflecting endoscopic severity by the UCEIS score was $20.8 \mu \mathrm{g} / \mathrm{g}$.Cr. On the other hand, the AUC of PEG-MUM and FIT in eMayo 0 versus 1 to 3 are 0.614 (95\% CI, 0.498-0.729) and 0.790 (95\% CI, $0.702-0.878$ ), respectively (data not shown), indicating that eMayo 0, 1 versus 2 and 3 was superior for the accurate evaluation of both biomarkers.

\section{Influence of long-term UC disease duration on urinary/ fecal biomarkers}

Finally, to investigate whether the UC disease duration affects the detection ability of PGE-MUM or FIT, we divided the patients into two groups (disease duration $<5$ years or $>5$ years) and performed a subgroup analysis of the endoscopic findings. The differences in characteristics between these two subgroups are shown in Table 3 . No significant differences in age as well as eMayo and UCEIS scores were observed between these two

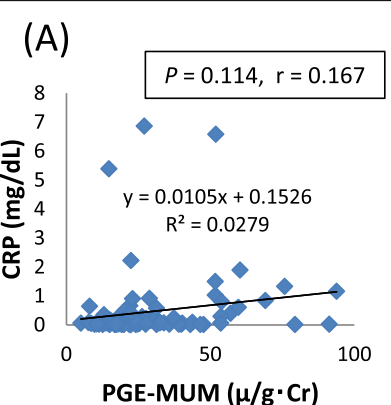

(B)

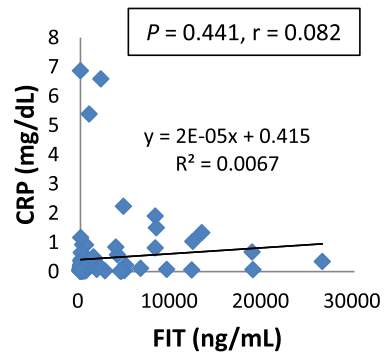

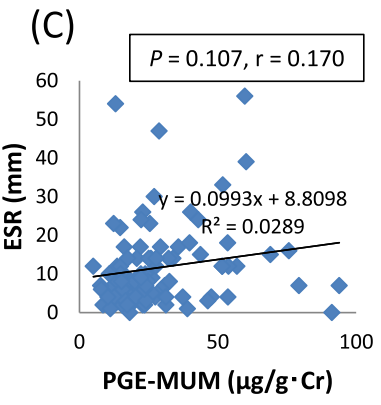

(D)

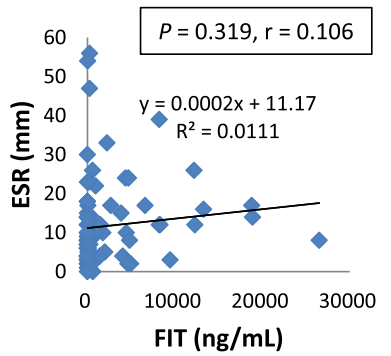

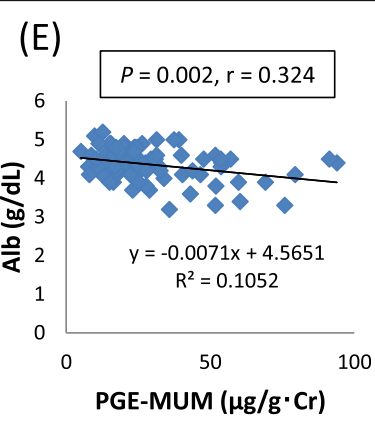

(F)

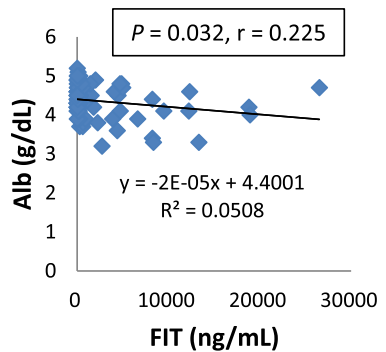

Fig. 1 Correlation between urinary/fecal biomarkers and blood biomarkers in patients with ulcerative colitis (UC). Scatter plots of (a) prostaglandin E-major urinary metabolite (PGE-MUM) vs. C-reactive protein (CRP), (b) fecal immunochemical occult blood test (FIT) vs. CRP, (c) PGE-MUM vs. erythrocyte sedimentation rate (ESR), (d) FIT vs. ESR, (e) PGE-MUM vs. serum concentration (Alb), and (f) FIT vs. Alb 

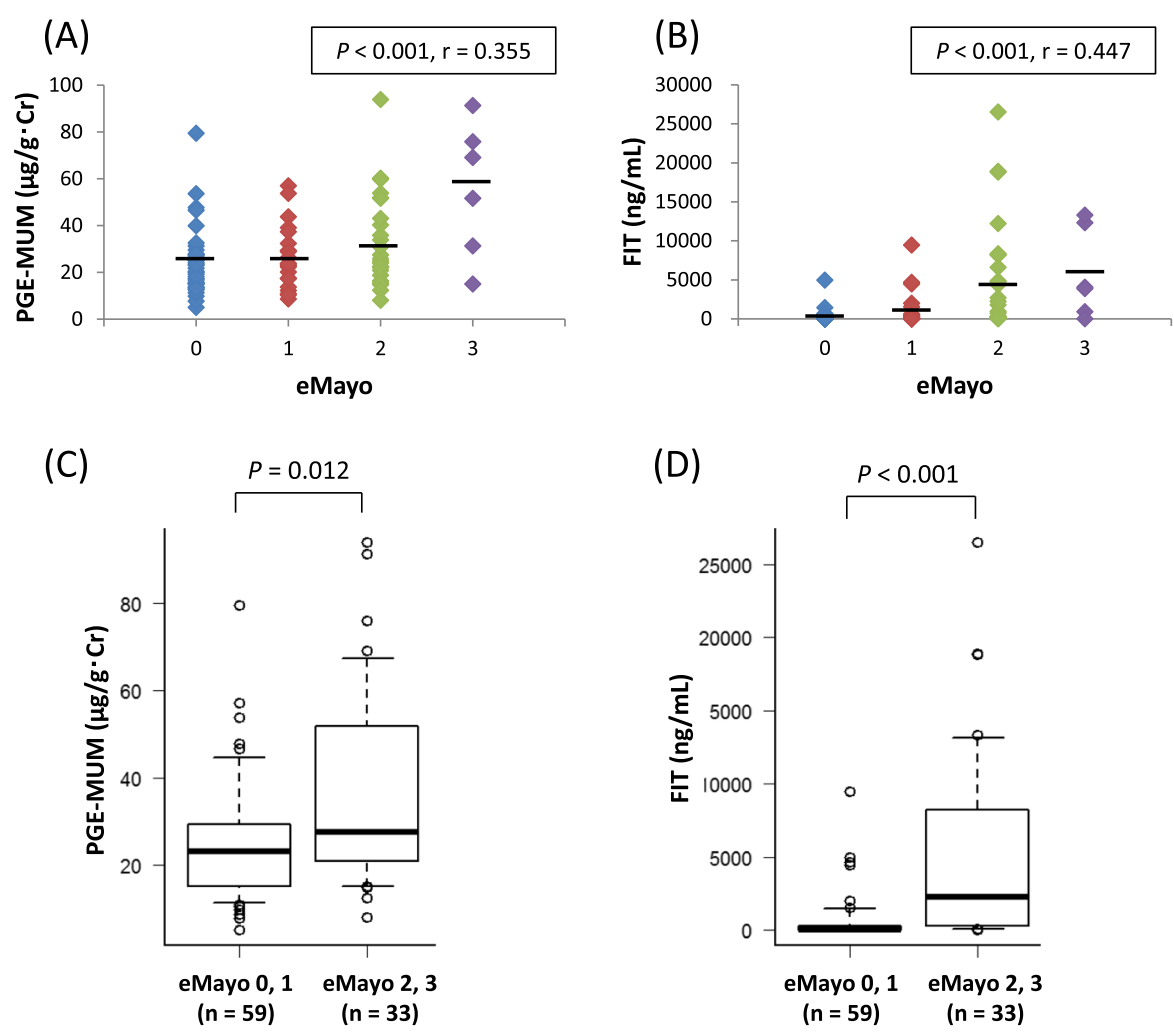

$(E)$

(F)
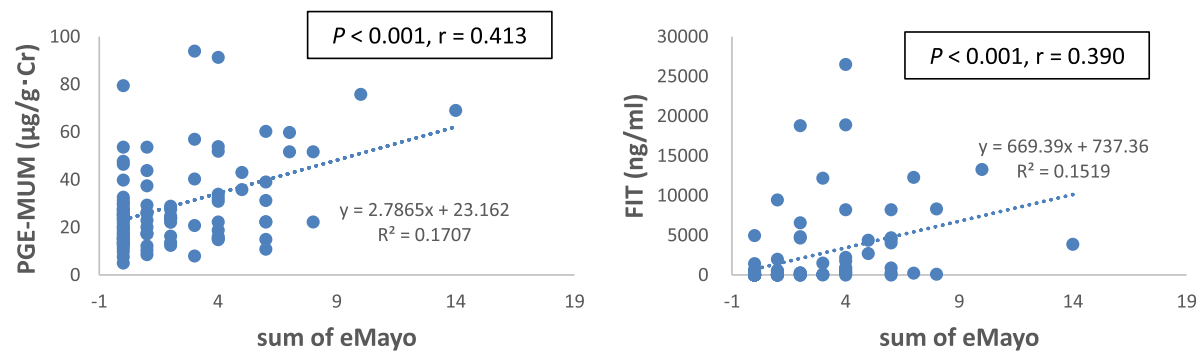

Fig. 2 Correlation between urinary/fecal biomarkers and Mayo endoscopic subscore (eMayo) in patients with ulcerative colitis (UC). Scatter plots of (a) eMayo vs prostaglandin E-major urinary metabolite (PGE-MUM), and (b) eMayo vs fecal immunochemical occult blood test (FIT). c Comparison of PGE-MUM level between subgroups (eMayo $\leq 1$ and eMayo $\geq 2$ ). $\mathbf{d}$ Comparison of FIT level between groups with eMayo $\leq 1$ and eMayo $\geq 2$. Scatter plots between (e) the sum of eMayo and the prostaglandin E-major urinary metabolite (PGE-MUM), and (f) the sum of eMayo and the fecal immunochemical occult blood test (FIT)

subgroups. The disease duration of the group with patients that were affected for $<5$ years was significantly higher than in those affected for $\geq 5$ years. CAI and the FIT value of the group with disease duration of $<5$ years were also significantly higher than that of patients with a disease duration of $\geq 5$ years.

We found significant positive correlations between PGE-MUM and eMayo in both groups $(<5$ years: $r=$ $0.296, P=0.041 ; \geq 5$ years: $r=0.376, P=0.012$, respectively) (Figs. $4 \mathrm{a}$ and $5 \mathrm{~b}$ ).

Although there was a significant positive correlation between FIT and eMayo in the group with a disease duration of $<5$ years $(r=0.569, P<0.001)$ (Fig. $4 \mathrm{c})$, no correlation was seen in the group with a disease duration of $\geq 5$ years $(r=0.251, P=0.101)$ (Fig. 5d). Next, we divided the endoscopic findings into eMayo 0, 1 and eMayo 2, 3 subgroups.

Although the group with a disease duration of $<5$ years had a significant difference of PGE-MUM between eMayo of 0 or 1 and eMayo of 2 or $3(P<0.001)$ (Fig. $5 b)$, there was no significant difference in the same group $(P=0.225)$ (Fig. 5a). Interestingly, in the group with a disease duration of $\geq 5$ years, FIT values did not differ significantly between the eMayo 0, 1 and eMayo 2, 3 subgroups (Fig. 5d). On the other hand, the PGE-MUM values tended to be higher in 

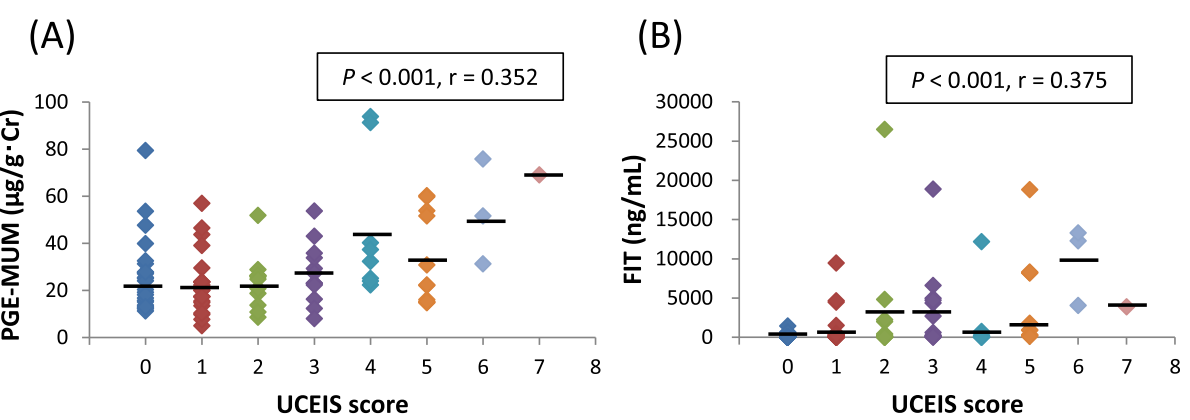

(C)

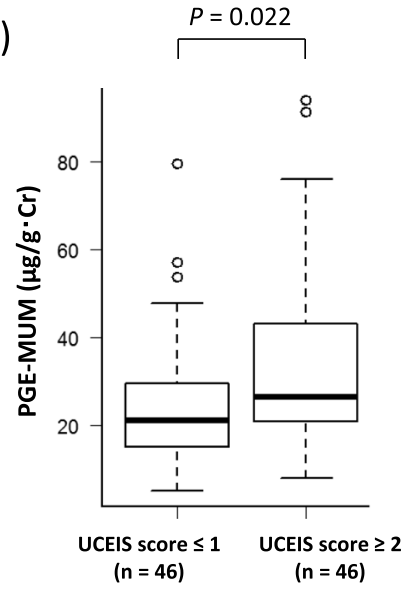

(D)

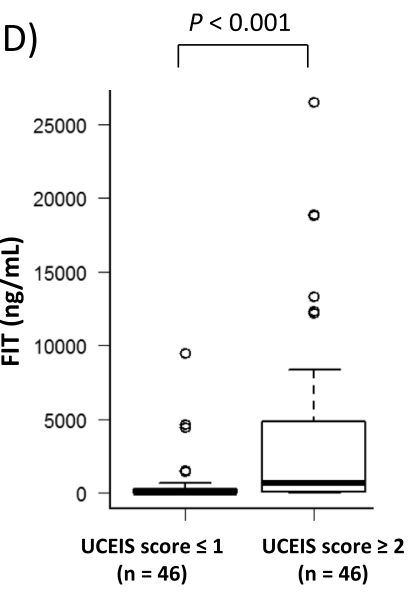

Fig. 3 Correlation between urinary/fecal biomarkers and the Ulcerative Colitis Endoscopic Index of Severity (UCEIS) in patients with ulcerative colitis (UC). Scatter plots of (a) prostaglandin E-major urinary metabolite (PGE-MUM) vs. UCEIS score, and (b) fecal immunochemical occult blood test (FIT) vs. UCEIS score. c Comparison of PGE-MUM level between subgroups (UCEIS score $\leq 1$ and UCEIS score $\geq 2$ ). $\mathbf{d}$ Comparison of FIT level between groups with UCEIS score $\leq 1$ and UCEIS score $\geq 2$

the eMayo 2, 3 subgroup than in the eMayo 0, 1 subgroup, but the inergroup differences were not significant (Fig. 5c).

We also examined significant differences between PGEMUM or FIT and the eMayo 0 and eMayo 1 to 3 groups. In all the cases, the PGE-MUM or FIT value was

Table 2 Test characteristics of two biomarker levels to predict endoscopic remission

\begin{tabular}{|c|c|c|c|c|}
\hline & \multicolumn{2}{|l|}{ eMayo } & \multicolumn{2}{|l|}{ UCEIS score } \\
\hline & PGE-MUM & FIT & PGE-MUM & FIT \\
\hline Cut-off value & $30.9 \mu \mathrm{g} / \mathrm{g} \cdot \mathrm{Cr}$ & $742 \mathrm{ng} / \mathrm{mL}$ & $20.8 \mu \mathrm{g} / \mathrm{g} \cdot \mathrm{Cr}$ & $31 \mathrm{ng} / \mathrm{mL}$ \\
\hline AUC & 0.644 & 0.826 & 0.636 & 0.789 \\
\hline $95 \% \mathrm{Cl}$ & $0.523-0.765$ & $0.74-0.912$ & $0.522-0.75$ & $0.698-0.879$ \\
\hline PPV & $55.2 \%$ & $81.2 \%$ & $60.3 \%$ & $70.2 \%$ \\
\hline NPV & $73.0 \%$ & $75.0 \%$ & $67.6 \%$ & $82.9 \%$ \\
\hline Sensitivity & $48.5 \%$ & $63.6 \%$ & $76.1 \%$ & $87.0 \%$ \\
\hline Specificity & $78.0 \%$ & $88.1 \%$ & $50.0 \%$ & $63.0 \%$ \\
\hline Accuracy & $67.4 \%$ & $79.3 \%$ & $63.0 \%$ & $75.0 \%$ \\
\hline
\end{tabular}

eMayo Mayo endoscopic subscore, UCEIS Ulcerative Colitis Endoscopic Index of Severity, PGE-MUM Prostaglandin E-Major Urinary Metabolite, FIT fecal immunochemical occult blood test, $A U C$ area under curve, $\mathrm{Cl}$ confidence interval, PPV Positive-predictive value, NPV negative-predictive value significantly different between eMayo 0 versus 1 to $3(P=$ $0.027, P<0.001$, respectively; data not shown). No significant difference in PGE-MUM was found between the eMayo 0 and eMayo 1 to 3 groups in both the $<5$ - and $\geq$ 5 -year groups $(P=0.355$ and $P=0.118$, respectively). The value of FIT was significantly different between eMayo 0 and eMayo 1 to 3 in the group with a disease duration of $<5$ years $(P<0.001)$. On the other hand, in the group with a disease period of $\geq 5$ years, the PGE-MUM value showed no significant difference between eMayo 0 and eMayo 1 to $3(P=0.070$; data not shown).

\section{Discussion}

The release of prostaglandin E 2 (PGE 2), a major chemical mediator involved in promoting and suppressing inflammation, is reportedly increased in the mucosa of UC patients versus healthy subjects [15]. Since PGE2 is produced at the site of inflammation and metabolized and decomposed upon its release into the blood, taking direct measurements is difficult. However, PGE-MUM, a urinary metabolite of PGE 2, is known to stabilize and reflect systemic PGE 2 production. In fact, although Arai et al. reported that PGE-MUM reflects mucosal 
Table 3 Difference of characteristics between the study subgroups

\begin{tabular}{|c|c|c|c|}
\hline \multirow[t]{2}{*}{ Characteristics } & \multicolumn{2}{|l|}{ Disease duration } & \multirow[t]{2}{*}{$P$ value } \\
\hline & $<5$ years, $n=48$ & $\geq 5$ years, $n=44$ & \\
\hline Age (year), mean $\pm S D$ & $46.6 \pm 15.2$ & $50.7 \pm 17.8$ & 0.232 \\
\hline Male/Female, n (\%) & $33 / 15(68.8 / 31.2)$ & $31 / 13(70.5 / 29.5)$ & \\
\hline Disease duration (year), mean $\pm S D$ & $2.1 \pm 1.3$ & $12.7 \pm 6.5$ & $<0.001$ \\
\hline \multicolumn{4}{|l|}{ Disease extent, n (\%) } \\
\hline Extensive colitis & $31(64.6)$ & $26(59.1)$ & \\
\hline Left sided colitis & $8(16.7)$ & $16(36.4)$ & \\
\hline Proctitis & $9(18.7)$ & $2(4.5)$ & \\
\hline CAl (Lichtiger's socre) mean \pm SD & $2.5 \pm 2.9$ & $1.3 \pm 1.7$ & 0.016 \\
\hline eMayo mean \pm SD & $1.1 \pm 1.0$ & $0.9 \pm 1.0$ & 0.250 \\
\hline eMayo 0, n (\%) & $16(33.3)$ & $22(50.0)$ & \\
\hline eMayo 1, n (\%) & $13(27.1)$ & $8(18.2)$ & \\
\hline eMayo 2, n (\%) & $16(33.3)$ & $11(25.0)$ & \\
\hline eMayo 3, n (\%) & $3(6.2)$ & $3(6.8)$ & \\
\hline UCEIS score mean \pm SD & $2.4 \pm 1.9$ & $1.8 \pm 1.9$ & 0.146 \\
\hline PGE-MUM $\left(\mu \cdot \mathrm{g}^{-1} \cdot \mathrm{Cr}^{-1}\right)$ mean $\pm \mathrm{SD}$ & $32.3 \pm 16.8$ & $25.8 \pm 19.5$ & 0.088 \\
\hline FIT (ng/ml), n (\%) mean \pm SD & $3285.8 \pm 5953.5$ & $969.6 \pm 2118.1$ & 0.017 \\
\hline \multicolumn{4}{|l|}{ Medication at study, n (\%) } \\
\hline Oral 5-ASA & $25(52.1)$ & $33(75)$ & \\
\hline Suppository 5-ASA & $10(20.8)$ & $7(15.9)$ & \\
\hline Systemic steroids & $9(18.8)$ & $3(6.8)$ & \\
\hline Azathioprine/Mercaptopurine & $22(45.8)$ & $13(29.5)$ & \\
\hline Tacrolimus & $7(14.6)$ & $3(6.8)$ & \\
\hline Biologics & $13(27.1)$ & $12(27.3)$ & \\
\hline
\end{tabular}

CAI clinical activity index, eMayo Mayo endoscopic subscore, UCEIS Ulcerative Colitis Endoscopic Index of Severity, PGE-MUM Prostaglandin E-Major Urinary Metabolite, FIT fecal immunochemical occult blood test, 5-ASA 5-aminosalicylic acid

inflammation severity in UC, it remains unclear whether PGE-MUM accurately reflects endoscopic severity either immediately after onset or in long-term patients [9]. Our study revealed that PGE-MUM was significantly correlated with endoscopic severity, even in patients with a disease duration $\geq 5$ years. The usefulness of biomarkers such as fecal calprotectin and FIT for IBD was recently reported. Although fecal examinations are non-invasive, feces have the possibility of directly reflecting inflammatory changes of the colonic mucosa and may pick up latent inflammation earlier, leading to the identification of several problems. First, due to heterogeneity, results may vary depending on the site of sample collection.

The value of FC is reportedly wide, even in the same stool sample collected on the same day [16]. Moreover, patients may find it cumbersome to bring stool samples from home to the hospital. On the other hand, urine specimens can be collected at the hospital and have the advantage of being more convenient and non-invasive. These are great benefits for pediatric patients with UC who experience difficulty undergoing frequent invasive endoscopic examinations. Hagiwara et al. reported that PGE-MUM is useful for pediatric patients with UC to evaluate their disease activity [17].

Here we compared the usefulness of PGE-MUM to that of FIT using urine samples. FIT, which is reportedly useful as a biomarker for patients with UC, is less expensive than other fecal biomarkers such as FC, can be performed at many facilities, and boasts immediately available results. Although FC requires $5-10 \mathrm{~g}$ of fecal material, FIT can be examined using only smaller samples $[18,19]$. FC reflects the volume of inflammatory cells in the intestinal tract, while FIT reflects blood originating from the damaged mucosa. PGE-MUM is similar to FC from the point of view of such an inflammation-derived mechanism. Although current reports show that FIT and FC can efficiently predict mucosal healing of UC, FIT was more sensitive than FC for predicting only eMayo 0 [20]. For the above reasons, we selected FIT rather than FC for comparison with PGEMUM. Ideal biomarkers are able to detect the recurrence of intestinal mucosal inflammation in an asymptomatic 

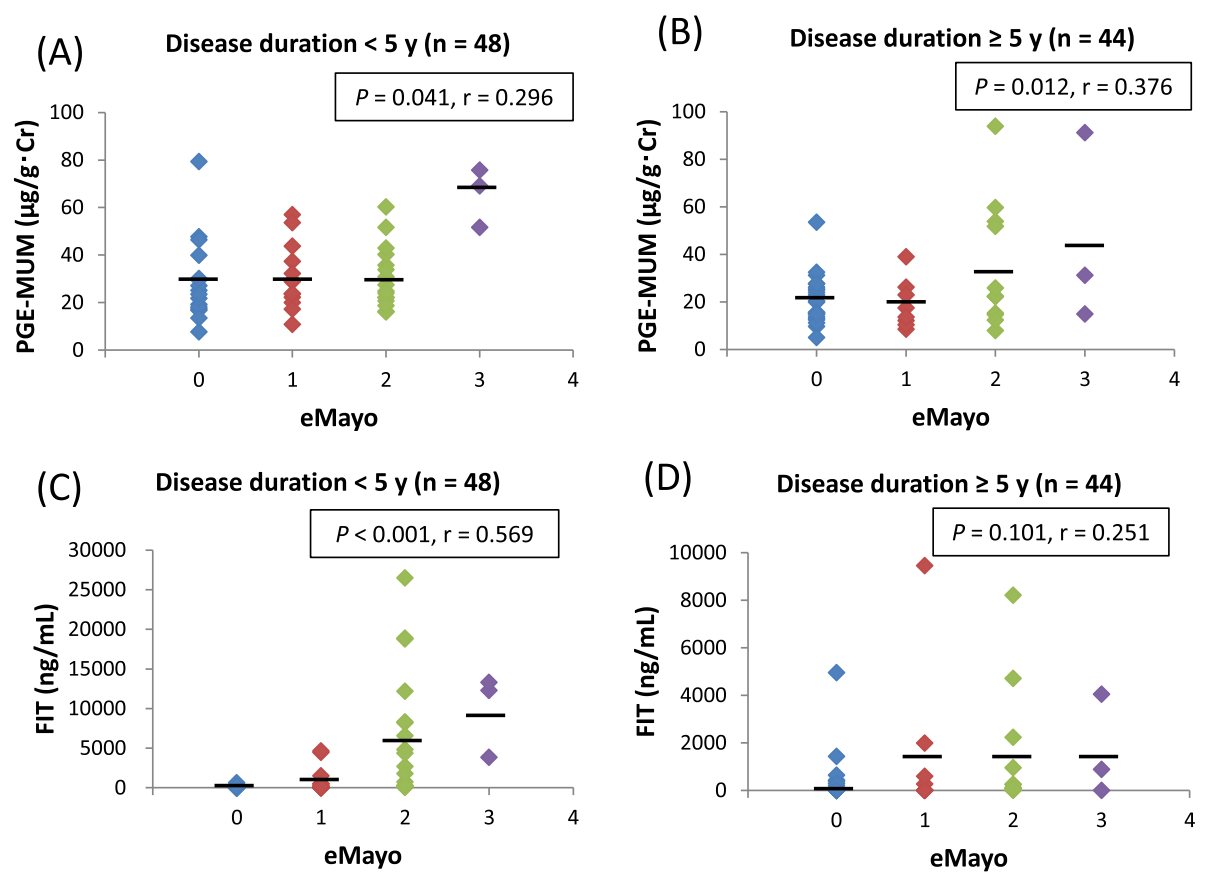

Fig. 4 Influence of a long disease duration of ulcerative colitis (UC) on the correlation between urinary/fecal biomarkers and Mayo endoscopic subscore (eMayo). Scatter plots of (a) eMayo vs. prostaglandin E-major urinary metabolite (PGE-MUM), and (b) eMayo vs. fecal immunochemical occult blood test (FIT) in patients with UC with disease duration of $<5$ years. Scatter plots of (c) eMayo vs. PGE-MUM and (d) eMayo vs. FIT in patients with UC with a disease duration of $\geq 5$ years

state. In our study, PGE-MUM and FIT were correlated with endoscopic severity and differed significantly between patients with and those without endoscopic activity. Considering its simplicity and minimal invasiveness, PGEMUM seems clinically useful. From our results, neither PGE-MUM nor FIT was correlated with CRP or ESR, blood biomarkers reflecting mucosal inflammation. However, it is interesting to note that serum Alb, a biomarker representing nutritional status, has a significant negative correlation with both PGE-MUM and FIT. Few reports show correlations between serum Alb and PGE-MUM or serum Alb and FIT, and the mechanism by which serum Alb or nutritional status affects the values of PGE-MUM and FIT is unknown. However, when the serum Alb value deviates significantly from normal or is abnormally low, caution may be required when evaluating PGE-MUM and FIT as biomarkers of UC activity.

The PGE-MUM values at baseline (eMayo 0 score), which are shown in Fig. 2a, were relatively higher than those published in a previous report. In a study by Arai et al., the mean PGE-MUM value was $15.9 \mu \mathrm{g} / \mathrm{g} \cdot \mathrm{Cr}$ in the eMayo 0 group, whereas in this study, it was $24.3 \mu \mathrm{g} / \mathrm{g} \cdot \mathrm{Cr}$ [9]. Laxative administration has been reported to increase the PGE-MUM value [21]. As almost all the enrolled patients were administered laxatives 1 day before the examination, the mean PGE-MUM value in this study may have been higher than that in a previous study.
PGE-MUM and FIT showed a significant positive correlation with eMayo in the group of patients with a disease duration of $<5$ years. Although PGE-MUM and eMayo were significantly correlated in patients with a disease duration of $\geq 5$ years, FIT was not correlated with eMayo in that group. In the group of patients with a disease duration of $<5$ years, FIT was significantly higher in the eMayo 2, 3 subgroup than in the eMayo 0, 1, but there was no significant difference between FIT and eMayo in the group with a disease duration of $\geq 5$ years. On the other hand, regarding PGE-MUM, no significant differences were seen between the eMayo 0,1 and eMayo 2, 3 subgroups in patients with a disease duration of < 5 years, but PGE-MUM tended to be higher in eMayo 2, 3 than in eMayo 0, 1 in patients with a disease duration $\geq 5$ years $(P=0.054)$. Patients with a long disease duration often experience repeated relapse and remission, and there are many sites of scarring and rough-surfaced mucosa in their intestinal tract. In these patients, the degree of bleeding is small although endoscopic abnormalities are recognized. Since FIT reflects the amount of bleeding itself, it may be difficult to reflect endoscopic abnormalities associated with inflammation in long-term diseased patients. On the other hand, since PGE-MUM reflects intestinal tract inflammation, it is likely able to reflect endoscopic abnormalities associated with inflammation in UC patients with long disease duration. Our results show that PGE- 
(A)

Disease duration $<5$ y $(n=48)$

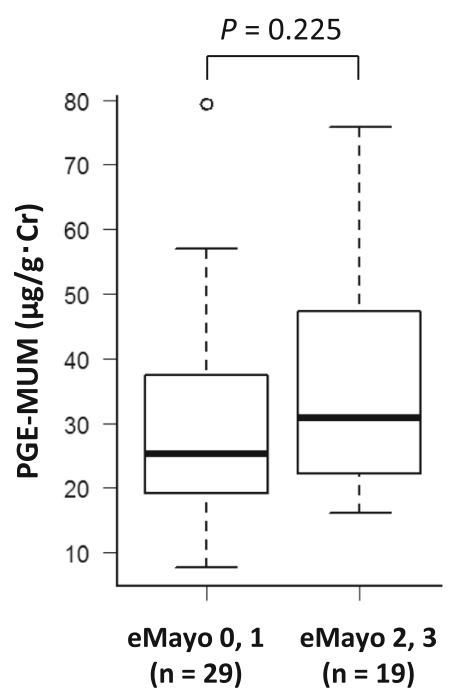

(C)

Disease duration $<5$ y $(n=48)$

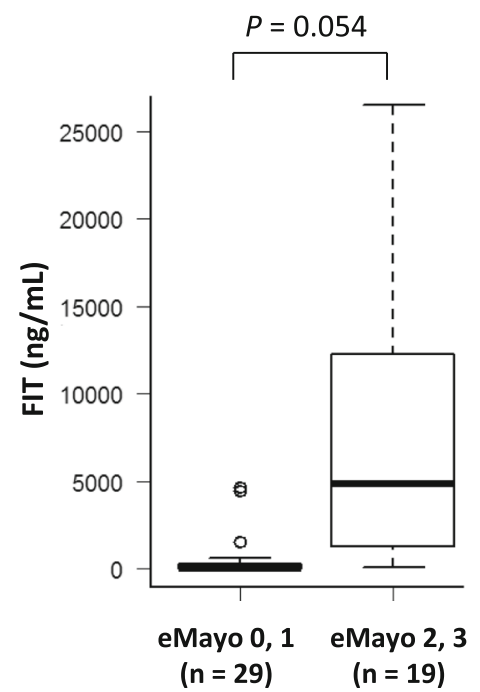

(B) Disease duration $\geq 5$ y $(n=44)$

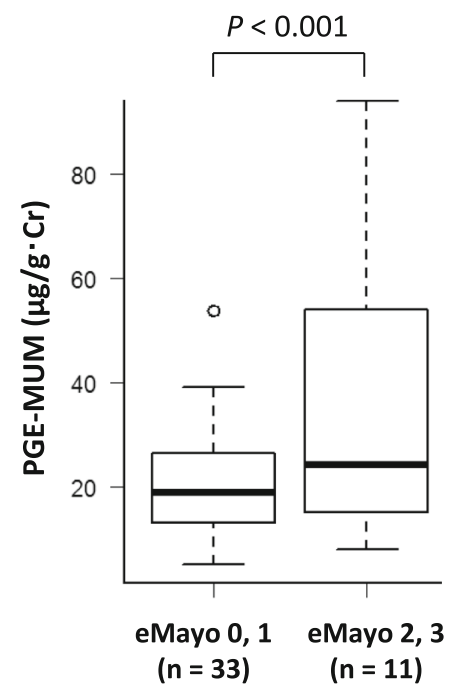

(D) Disease duration $\geq 5$ y $(n=44)$

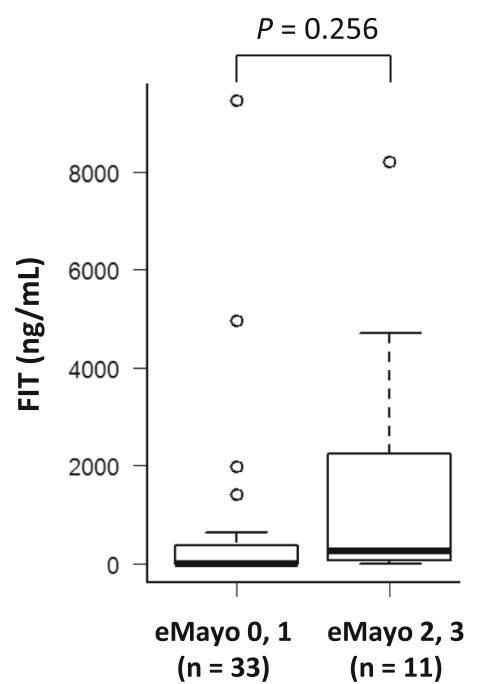

Fig. 5 Influence of a long disease duration of ulcerative colitis (UC) on a comparison of urinary/fecal biomarkers between subgroups (Mayo endoscopic subscore [eMayo] $\leq 1$ and eMayo $\geq 2$ ). Comparison of (a) prostaglandin E-major urinary metabolite (PGE-MUM) and (c) fecal immunochemical occult blood test (FIT) levels between groups with an eMayo $\leq 1$ and eMayo $\geq 2$ in patients with UC with a disease duration of $<5$ years. Comparison of PGE-MUM (b) and FIT (d) levels between the groups with an eMayo $\leq 1$ and eMayo $\geq 2$ in patients with UC with a disease duration $>5$ years

MUM may be a more useful biomarker for predicting endoscopic severity when disease duration is prolonged; however, the accumulation of cases is necessary to confirm these results.

There are some limitations to this study. First, it was performed in a single center and included a small number of patients. Also, in this study, it was not proven whether PGE-MUM can predict UC recurrence. To clarify that point, there is a need for periodic measurements of PGE-MUM to be performed in the same patient and prospective follow-up be performed of patients with relapse. In recent years, treatments aimed at histological healing more advanced than mucosal healing have also been required, and reports of the comparison between histological healing and biomarkers have been found [22-25]. Further studies are needed to determine if PGE-MUM could be a biomarker to predict histologic healing.

\section{Conclusion}

PGE-MUM is a biomarker capable of predicting endoscopic activity as well as FIT and could be more sensitive 
than FIT to represent the endoscopic UC activity in long-term affected patients. Further studies are expected to effectively utilize PGE-MUM measurements to maintain mucosal healing in UC patients.

\section{Abbreviations}

UC: Ulcerative colitis; CRP: C-reactive protein; FC: Fecal calprotectin; FIT: Fecal immunochemical occult blood test; PGE-MUM: Prostaglandin E-major urinary metabolite; CAl: Clinical activity index; eMayo: Mayo endoscopic subscore; UCEIS: Ulcerative Colitis Endoscopic Index of Severity; ESR: Erythrocyte sedimentation rate; Alb: Albumin; ROC: Receiver-operating characteristic; AUC: Area under the curve; Cl: Confidence interval

\section{Acknowledgements}

Not applicable.

\section{Authors' contributions}

$\mathrm{NI}$ contributed to this work; KS and TM designed the research study; RT, ST and TK collected the data; ST2, MY MI and YH analyzed the data; NI and KS wrote the paper; SO, TF and HM provided critical insight regarding paper preparation. All authors have read and approved the manuscript.

\section{Funding}

This study was funded by a Grant-in-Aid for Scientific Research (C) (no. 18 K07908) from the Japanese Ministry of Education, Culture, Sports, Science, and Technology. The funders had a role in study design, decision to publish and preparation of the manuscript. No additional external funding was received for this study.

\section{Availability of data and materials}

Not applicable.

\section{Ethics approval and consent to participate}

All study participants provided verbal consent. The reason: Since the samples used in this study were urine and feces, Participants will not be harmed or lost. They are willing to provide samples and the Institutional Review Board of Hamamatsu University School of Medicine reviewed and approved all procedures (including verbal consent) for the experimental specimens.

\section{Consent for publication}

Not applicable.

\section{Competing interests}

The authors declare that they have no competing interests.

\section{Author details}

'First Department of Medicine, Hamamatsu University School of Medicine, 1-20-1 Handayama, Higashi-ku, Hamamatsu 431-3192, Japan. ${ }^{2}$ Department of Endoscopic and Photodynamic Medicine, Hamamatsu University School of Medicine, Hamamatsu, Shizuoka, Japan. ${ }^{3}$ Department of Laboratory Medicine, Hamamatsu University School of Medicine, Hamamatsu, Shizuoka, Japan. ${ }^{4}$ Center for Clinical Research, Hamamatsu University School of Medicine, Hamamatsu, Shizuoka, Japan.

Received: 3 October 2019 Accepted: 31 March 2020

Published online: 19 April 2020

\section{References}

1. Podolsky DK. Inflammatory bowel disease. N Engl J Med. 2002;347:417-29.

2. Langholz E, Munkholm P, Davidsen M, Binder V. Course of ulcerative colitis: analysis of changes in disease activity over years. Gastroenterology. 1994; 107:3-11

3. Colombel JF, Rutgeerts P, Reinisch W, Esser D, Wang Y, Lang Y, et al. Early mucosal healing with infliximab is associated with improved long-term clinical outcomes in ulcerative colitis. Gastroenterology. 2011;141:1194-201.

4. Vermeire S, Van Assche G, Rutgeerts P. C-reactive protein as a marker for inflammatory bowel disease. Inflamm Bowel Dis. 2004;10:661-5.

5. Sands BE. Biomarkers of inflammation in inflammatory bowel disease. Gastroenterology. 2015;149:1275-85.
6. Schoepfer AM, Beglinger C, Straumann A, Trummler M, Renzulli P, Seibold F. Ulcerative colitis: correlation of the Rachmilewitz endoscopic activity index with fecal calprotectin, clinical activity, C-reactive protein, and blood leukocytes. Inflamm Bowel Dis. 2009:15:1851-8.

7. D'Haens G, Ferrante M, Vermeire S, Baert F, Noman M, Moortgat L, et al. Fecal calprotectin is a surrogate marker for endoscopic lesions in inflammatory bowel disease. Inflamm Bowel Dis. 2012;18:2218-24.

8. Yamamoto T, Shimoyama T, Umegae S, Matsumoto K. Endoscopic score vs fecal biomarkers for predicting relapse in patients with ulcerative colitis after clinical remission and mucosal healing. Clin Transl Gastroenterol. 2018; 9:e136.

9. Arai Y, Arihiro S, Matsuura T, Kato T, Matsuoka M, Saruta M, et al. Prostaglandin E-major urinary metabolite as a reliable surrogate marker for mucosal inflammation in ulcerative colitis. Inflamm Bowel Dis. 2014;20: 1208-16.

10. Okayasu I, Ohnishi H, Sarandi I, Shojima J, Komatsu J, Oritsu M. Significant increase of prostaglandin $\mathrm{E}$ major urinary metabolite in male smokers a screening study of age and gender differences using a simple radioimmunoassay. J Clin Lab Anal. 2014;28:32-41.

11. Horikiri T, Hara H, Saito N, Araya J, Takasaka N, Utsumi H. Increased levels of prostaglandin E major urinary metabolite (PGE MUM) in chronic fibrosing interstitial pneumonia. Respir Med. 2017:122:43-50.

12. Lichtiger S, Present DH. Preliminary report: Cyclosporin in treatment of severe active ulcerative colitis. Lancet. 1990:336:16-9.

13. Schroeder KW, Tremaine WJ, Ilstrup DM. Coated oral 5-aminosalicylic acid therapy for mildly to moderately active ulcerative colitis. A randomized study. N Engl J Med. 1987;317:1625-9.

14. D'Haens G, Sandborn WJ, Feagan BG, Geboes K, Hanauer SB, Irvine EJ, et al. A review of activity indices and efficacy end points for clinical trials of medical therapy in adults with ulcerative colitis. Gastroenterology. 2007;132: 763-86.

15. Raab Y, Sundberg C, Hällgren R, Knutson L, Gerdin B. Mucosal synthesis and release of prostaglandin E2 from activated eosinophils and macrophages in ulcerative colitis. Am J Gastroenterol. 1995;90:614-20.

16. Lasson A, Stotzer PO, Öhman L, Isaksson S, Sapnara M, Strid H. The intraindividual variability of faecal calprotectin: a prospective study in patients with active ulcerative colitis. J Crohns Colitis. 2015:9:26-32.

17. Hagiwara SI, Okayasu I, Fujiwara M, Matsuura M, Ohnishi H, Ito S, et al. Prostaglandin E-major urinary metabolite as a biomarker for pediatric ulcerative colitis activity. J Pediatr Gastroenterol Nutr. 2017;64:955-61.

18. Costa F, Mumolo MG, Bellini M, Romano MR, Ceccarelli L, Arpe P, et al. Role of faecal calprotectin as non-invasive marker of intestinal inflammation. Dig Liver Dis. 2003;35:642-7.

19. Vilkin A, Rozen P, Levi Z, Waked A, Maoz E, Birkenfeld S, et al. Performance characteristics and evaluation of an automated developed and quantitative, immunochemical, fecal occult blood screening test. Am J Gastroenterol. 2005;100:2519-25

20. Takashima S, Kato J, Hiraoka S, Nakarai A, Takei D, Inokuchi T, et al. Evaluation of mucosal healing in ulcerative colitis by fecal calprotectin vs. fecal immunochemical test. Am J Gastroenterol. 2015;110:873-80.

21. Fujiwara M, Okayasu I, Oritsu M, et al. Significant increase in prostaglandin E-main urinary metabolite by laxative administration: comparison with ulcerative colitis. Digestion. 2000;61(3):201-6.

22. Mak WY, Buisson A, Andersen MJ Jr, Lei D, Pekow J, Cohen RD, et al. Fecal calprotectin in assessing endoscopic and histological remission in patients with ulcerative colitis. Dig Dis Sci. 2018;63:1294-301.

23. Narang V, Kaur R, Garg B, Mahajan R, Midha V, Sood N, et al. Association of endoscopic and histological remission with clinical course in patients of ulcerative colitis. Intest Res. 2018:6:55-61.

24. Ponte A, Pinho R, Fernandes S, Rodrigues A, Alberto L, Silva JC, et al. Impact of histological and endoscopic remissions on clinical recurrence and recurrencefree time in ulcerative colitis. Inflamm Bowel Dis. 2017;23:2238-44.

25. Bryant RV, Burger DC, Delo J, Walsh AJ, Thomas S, von Herbay A, et al. Beyond endoscopic mucosal healing in UC: histological remission bette predicts corticosteroid use and hospitalization over 6 years of follow-up. Gut. 2016:65:408-14

\section{Publisher's Note}

Springer Nature remains neutral with regard to jurisdictional claims in published maps and institutional affiliations. 